\title{
Diversidad, abundancia y distribución de la macrofauna bentónica de las costas rocosas al norte del Estado Sucre, Venezuela
}

\author{
Johanna Fernández ${ }^{1}$, Mayré Jiménez² \& Thays Allen² \\ 1. Museo del Mar, Universidad de Oriente, Venezuela; johnannafer@yahoo.com.ar \\ 2. Departamento de Biología Marina, Instituto Oceanográfico de Venezuela, Universidad de Oriente; \\ mayrej@gmail.com, thayscor@yahoo.com
}

Recibido 29-VII-2013. Corregido 15-I-2014. Aceptado 14-II-2014.

\begin{abstract}
Diversity, abundance and distribution of benthic macrofauna on rocky shores from North Sucre State, Venezuela. The rocky intertidal zone is among the most extreme physical environments on Earth. Organisms living in this area are constantly stricken by physico-chemical and biological factors. Due to the ecological importance of these areas, we studied the diversity, abundance and distribution of the rocky coastline benthic macrofauna, from the North coast of Sucre State, Venezuela. We performed bimonthly samplings from November 2008 to September 2009. The collection of biological material in the littoral zone (supra, mid and infralittoral) was done manually with a grid of $0.25 \mathrm{~m}^{2}$. Organisms were preserved in $10 \%$ formalin for later identification and analysis (ecological parameters and Kruskal-Wallis test to the abundance and diversity). We found a total 19020 organisms (86spp.), in 8 phyla, 45 families and 47 genera. Mollusks were the most abundant and diverse (58 spp.), followed by arthropods (12spp.), annelids ( $7 \mathrm{spp}$.$) , echinoderms ( 5 \mathrm{spp}$.), and the less represented cnidarians, sipunculids, nemertinids and urochordates (1sp.). The zonation found coincided with the universal scheme of zonation. The towns of Rio Boca and Rio Caribe presented the highest values of ecological parameters, and the lowest were found in Playa Grande. Statistical significant differences were found in the abundance and diversity of macrofauna among the three zones. The little information on the composition and distribution of macrobenthic rocky coastline, prevents a better comparison, however the results contribute to the knowledge of the marine biodiversity in this region. Rev. Biol. Trop. 62 (3): 947-956. Epub 2014 September 01.
\end{abstract}

Key words: benthic, rocky, coast line, ecology, diversity.

El litoral rocoso está constituido en su mayor parte por rocas sometidas a la acción de las olas y mareas. Representa uno de los ambientes físicamente más extremos de la tierra.

En las costas litorales rocosas, los diferentes organismos (invertebrados y vertebrados) que allí habitan, se han adaptado a estos ambientes, determinando una zonación o distribución de especies, en franjas o bandadas, según ciertos factores incidentes: físico-químicos (como el régimen climático, energía del viento, oleaje, intensidad lumínica, mareas, topografía del sustrato, salinidad y temperatura) y biológicos (presión por depredadores, competencia por espacio y alimento) (Dauer, 1993; Saiz, 1997). Consecuentemente, estos factores y la comunidad espacial condensada, han hecho de la zona intermareal un "laboratorio natural ideal" para estudiar el papel que tienen los organismos en la determinación de la abundancia y distribución en la naturaleza (Lewis, 1961; Paine, 1974).

En este sentido, los patrones de distribución de la macrofauna bentónica costera ha servido como herramienta básica para la identificación y delimitación de áreas biogeográficas definidas, por lo que, la evaluación de las comunidades marinas, se basa principalmente en estudios ecológicos que permiten determinar las especies integrantes, además de arrojar información valiosa sobre la estructura de las poblaciones ricas y abundantes que cohabitan 
en una zona determinada (Levinton, 1995; Margalef, 1995, INVEMAR, 2004).

Son varios los trabajos realizados sobre las comunidades bentónicas en diferentes zonas geográficas costeras en América y el Caribe, se mencionan, el de Lewis (1961) en Barbados; Brattström $(1980 ; 1985)$ en Colombia y Panamá, respectivamente, Vázquez \& Alonso (2004) en el Parque Nacional Fray Jorge, Chile. En Venezuela se encuentran los trabajos de Rodríguez (1959) en la Isla de Margarita, Urosa (1972), Quintana (1980), Sant \& De Grado (1997) al sur del Golfo de Cariaco; CAMUDOCA (2006), y Fernández y Jiménez (2007) en la costa norte del estado Sucre y Península de Paria. Igualmente, se encuentra el trabajo realizado por Cruz-Motta (2007) en la costa central y occidental del país.

En la actualidad, la potencial pérdida de biodiversidad marina, ha estimulado un aumento en el número de estudios para determinar su importancia en el funcionamiento de los ecosistemas, sirviendo como indicador del equilibrio entre la preservación y la extinción de algunas especies (Pachepsky, Crawford, Brow \& Squire, 2001). Hasta la fecha existen pocos trabajos tendientes a caracterizar los invertebrados bénticos de estos sistemas, presentándose inclusive zonas litorales en el país donde no existe ningún material publicado que haga referencia a su biota asociada, por lo que el objetivo de esta investigación fue estudiar la diversidad, abundancia y distribución de la macrofauna bentónica de las costas rocosas al norte del estado Sucre, Venezuela.

\section{MATERIALES Y MÉTODOS}

Área de estudio: El estado Sucre, se encuentra ubicado en la región nororiental de Venezuela, donde se escogieron cuatro localidades de naturaleza rocosa, ubicadas entre la ciudad de Carúpano y Río Caribe, con una extensión aproximada de $15 \mathrm{~km}$ : 1: Río Caribe $\left(10^{\circ} 42^{\prime} \mathrm{N}-63^{\circ} 06^{\prime} \mathrm{W}\right), 2$ : Boca de Río (1040' $\left.\mathrm{N}-63^{\circ} 13^{\prime} \mathrm{W}\right), 3$ : Playa Grande $\left(10^{\circ} 39^{\prime} \mathrm{N}\right.$

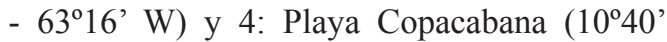

$\left.\mathrm{N}-63^{\circ} 18^{\prime} \mathrm{W}\right)$. Es una zona con incidencia de continuos vientos que originan un fuerte $y$ constante oleaje.

Toma de muestras y análisis de datos: Se realizaron muestreos bimensuales durante un año (noviembre 2008-septiembre 2009), cubriendo tanto la época de lluvia (de mayo a noviembre), como de sequía (de diciembre a abril), según Terejova, Quintero \& Piñero (1998). Se establecieron transectos perpendiculares a la costa, de $5 \mathrm{~m}$ de longitud, para cada localidad, siguiendo la metodología de Jones (1980), tomando como puntos de partida y final la posición de organismos indicadores de cada ambiente. A lo largo del transecto se fijaron un total de seis estaciones, con su respectiva réplica, ubicando las estaciones 1 y 2 en la zona supralitoral, las estaciones 3 y 4 en la zona mediolitoral, y las estaciones 5 y 6 en la zona infralitoral.

Se utilizó una cuadrícula metálica de $0.25 \mathrm{~m}^{2}$ para la recolecta de todos los organismos bentónicos, según metodología de Weinberg (1978) y Damianidis \& Chintiroglou (2000). Los organismos recolectados fueron colocados en frascos de vidrio y preservados en formalina al $10 \%$. La identificación se basó en la bibliografía disponible: moluscos (Abbott, 1974; 1994; Díaz \& Puyana, 1994; Abbott \& Morris, 1995); crustáceos (Granadillo, 1982; Lira, 1997; Martin \& Davis, 2001); esponjas (Zea, 1987; Ramírez, 1997); equinodermos (Belmonte, 1985; Herrera \& Betancourt, 2004) y poliquetos (Fauchald, 1977; Uebelacker et al., 1984; Salazar et al., 1988). Posteriormente, se determinó la abundancia de organismos y riqueza de especies (Margalef, 1995), diversidad con Shannon-Wienner, y equidad con Lloyd-Ghelardi. El análisis estadístico incluyó una prueba no paramétrica de Kruskal-Wallis a la abundancia y diversidad de los organismos durante el periodo de estudio. Además de un análisis de ANOSIM - MDS a la abundancia de la macrofauna bentónica entre zonas en las cuatro localidades. 


\section{RESULTADOS}

Se recolectaron un total de 19020 organismos, contenidos en 86 especies, distribuidos en ocho Phyla, 14 clases, 21 órdenes, 45 familias y 47 géneros. Los moluscos representaron el grupo más abundante y diverso con 18048 organismos y 58 especies, seguido de los artrópodos con 78 organismos y 12 especies, los anélidos con 89 organismos y 7 especies, equinodermos con 15 organismos y 5 especies, y por último, los cnidarios, sipuncúlidos, nemertinos y urocordados, que resultaron los menos abundantes con una especie cada uno (Cuadro 1 y Fig. 1).

El patrón de distribución de la macrofauna observada, corresponde a una sucesión de bandas, líneas y franjas horizontales, debido a la formación de zonas de choque, como consecuencia del fuerte y constante oleaje, donde se caracterizaron tres zonas. La supralitoral o zona de spray, representada por 30spp., siendo los más abundantes, los moluscos planáxidos y litorínidos, y los crustáceos balánidos y decápodos. La mediolitoral, caracterizada por el impacto directo del oleaje, representada por 69spp., entre los cuales se destacaron por abundancia los géneros: Planaxis, Mitrella, Isognomon, Perna, Brachidontes, Acanthopleura, Chiton, Balanus, Echinimetra y Ophioder$m a$; poliquetos (Nereididae y Sabellariidae), sipuncúlidos y nemertínidos. La infralitoral o zona completamente sumergida, caracterizada por 70spp., siendo los géneros Mitrella, Acmaea, Fissurella, Thais, Tegula, Perna, Brachidontes, Isognomon, Carditameras, Acanthopleura, Chiton, Balanus, Echinometra, Holothuria y Lytechinus; los poliquetos Sabellariidae, Serpulidae, Syllidae y Cirratulidae, los más abundantes.

Al considerar la abundancia entre las localidades, el valor más alto correspondió a Río Caribe (6 876 organismos), seguido de Playa Copacabana (4 396 organismos), Boca de Río (4 280 organismos) y Playa Grande (3 469 organismos), mientras que entre las zonas, en términos generales, la supralitoral presentó el valor más elevado, y la medio e infralitoral los valores más bajos de abundancia (Fig. 2).

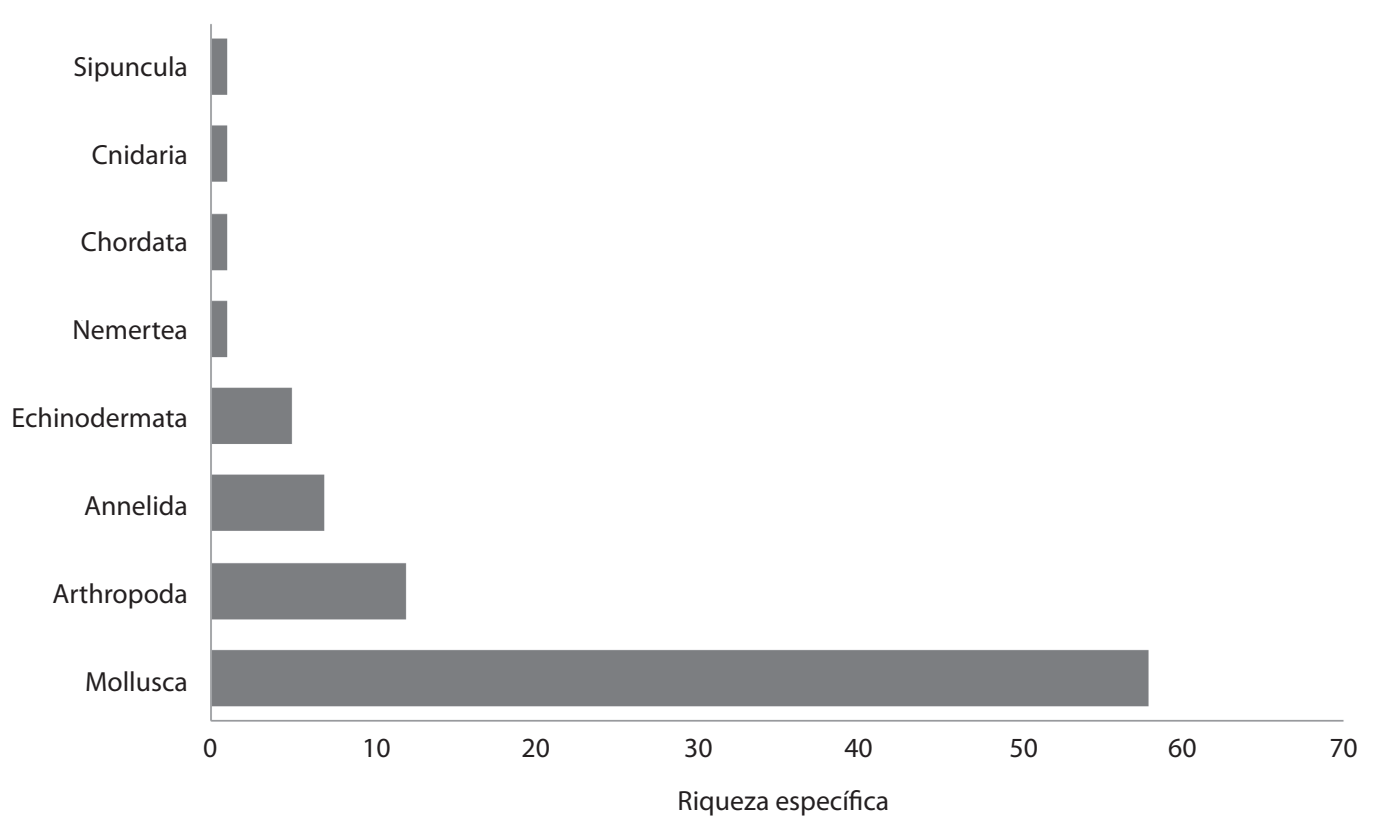

Fig. 1. Riqueza numérica de los diferentes filos que comprenden el macrobentos de la costa rocosa del estado Sucre.

Fig. 1. Richness of different Phyla of the macrobenthic on the rocky coast of Sucre State. 
CUADRO 1

Lista taxonómica de las especies de la macrofauna del litoral rocoso de las localidades estudiadas

TABLE 1

Taxonomic list of species of rocky shore macrofauna of the study areas

\begin{tabular}{|c|c|c|c|}
\hline Taxón & Especie & Taxón & Especie \\
\hline \multicolumn{2}{|c|}{ MOLLUSCA/ Gasteropoda } & Veneridae & Tivela mactroides \\
\hline \multirow[t]{2}{*}{ Fissurellidae } & Fissurella nimbosa & Psammobiidae & Asaphis deflorata \\
\hline & Diodora $\mathrm{sp}$. & Carditidae & Carditamera gracilis \\
\hline \multirow[t]{3}{*}{ Acmaeidae } & Acmaea antillarum & Corbulidae & Corbula dietziana \\
\hline & Acmaea leucopleura & Polyplacophora & \\
\hline & Acmaea pustulata & Ischnochitonidae & Ischnochiton striolatus \\
\hline \multirow[t]{2}{*}{ Trochidae } & Tegula fasciata & Chitonidae & Chiton marmoratus \\
\hline & Tegula excavata & & Chiton squamosus \\
\hline \multirow[t]{2}{*}{ Turbinidae } & Astraea tuber & & Chiton tuberculatus \\
\hline & Astraea caelata & & Acanthopleura granulata \\
\hline \multirow[t]{4}{*}{ Littorinidae } & Littorina interrupta & Callistoplacidae & Ceratozona squalida \\
\hline & Littorina ziczac & ARTROPODA/ Maxillopoda & \\
\hline & Littorina meleagris & Chthamalidae & Chthamalus fragilis \\
\hline & Littorina obtusata & Balanidae & Amphibalanus amphitrite \\
\hline Turritellidae & Turritella variegata & & Balanus trigonus \\
\hline \multirow[t]{2}{*}{ Planaxidae } & Planaxis lineatus & & Balanus venustus \\
\hline & Planaxis nucleus & & Balanus inexpectatus \\
\hline \multirow[t]{2}{*}{ Calyptraeidae } & Crepidula convexa & & Amphibalanus improvisus \\
\hline & Crepidula plana & Malacostraca & \\
\hline \multirow[t]{2}{*}{ Muricidae } & Chicoreus brevifrons & Porcellanidae & Petrolisthes armatus \\
\hline & Phyllonotus pomum & Xanthidae & Panopeus herbstii \\
\hline \multirow[t]{3}{*}{ Thaididae } & Stramonita haemastoma & & Eurypanopeus abbreviatus \\
\hline & Stramonita floridana & Grapsidae & Aratus pisonii \\
\hline & Thais rústica & & Amphipoda sp.1 \\
\hline Buccinidae & Pisania tincta & & Ermitaño sp.1 \\
\hline \multirow[t]{4}{*}{ Columbellidae } & Anachis sp. & ECHINODERMATA/ Echinoid & \\
\hline & Nitidella nitida & Echinometridae & Echinometra lucunter \\
\hline & Mitrella nycteis & & Echinometra viridis \\
\hline & Mitrella ocellata & Toxopneustidae & Lytechinus variegatus \\
\hline \multirow[t]{4}{*}{ Fasciolariidae } & Fasciolaria tulipa & Holothurioidea & \\
\hline & Latirus mcgintyi & Holothuriidae & Holothuria sp. \\
\hline & Leucozonia nassa & Ophiodermatidae & Ophioderma cinerea \\
\hline & Leucozonia ocellata & ANNELIDA/ Oligochaerta & \\
\hline \multicolumn{3}{|l|}{ Bivalvia } & Oligochaeta sp.1 \\
\hline Arcidae & Arca zebra & Polychaeta & \\
\hline \multirow[t]{7}{*}{ Mytilidae } & Perna perna & Sabellariidae & Sabellariidae sp.1 \\
\hline & Perna viridis & Sabellidae & Sabellidae sp.1 \\
\hline & Brachidontes exustus & Serpulidae & Serpulidae sp.1 \\
\hline & Brachidontes domingensis & Cirratulidae & Cirratulidae sp.1 \\
\hline & Brachidontes modiolus & Syllidae & Syllidae sp.1 \\
\hline & Lithophaga antillarum & Nereididae & Nereididae sp.1 \\
\hline & Modiolus squamosus & CNIDARIA/ Antozoa & \\
\hline Pteriidae & Pinctada imbricata & Zoanthidae & Zoanthus sp. \\
\hline \multirow[t]{3}{*}{ Isognomidae } & Isognomon alatus & SIPUNCULIDA/ Sipunculidea & \\
\hline & Isognomon radiatus & Sipunculidae & Sipunculus sp. \\
\hline & Isognomon bicolor & NEMERTINA/ Anopla & \\
\hline \multirow[t]{3}{*}{ Ostreidae } & Ostrea sp. & Lineidae & Lineidae sp.1 \\
\hline & Ostrea equestris & CHORDATA/ Ascidiacea & \\
\hline & Crassostrea sp. & Ascidiidae & Phallusia nigra \\
\hline Lucinidae & Codakia sp. & & \\
\hline
\end{tabular}



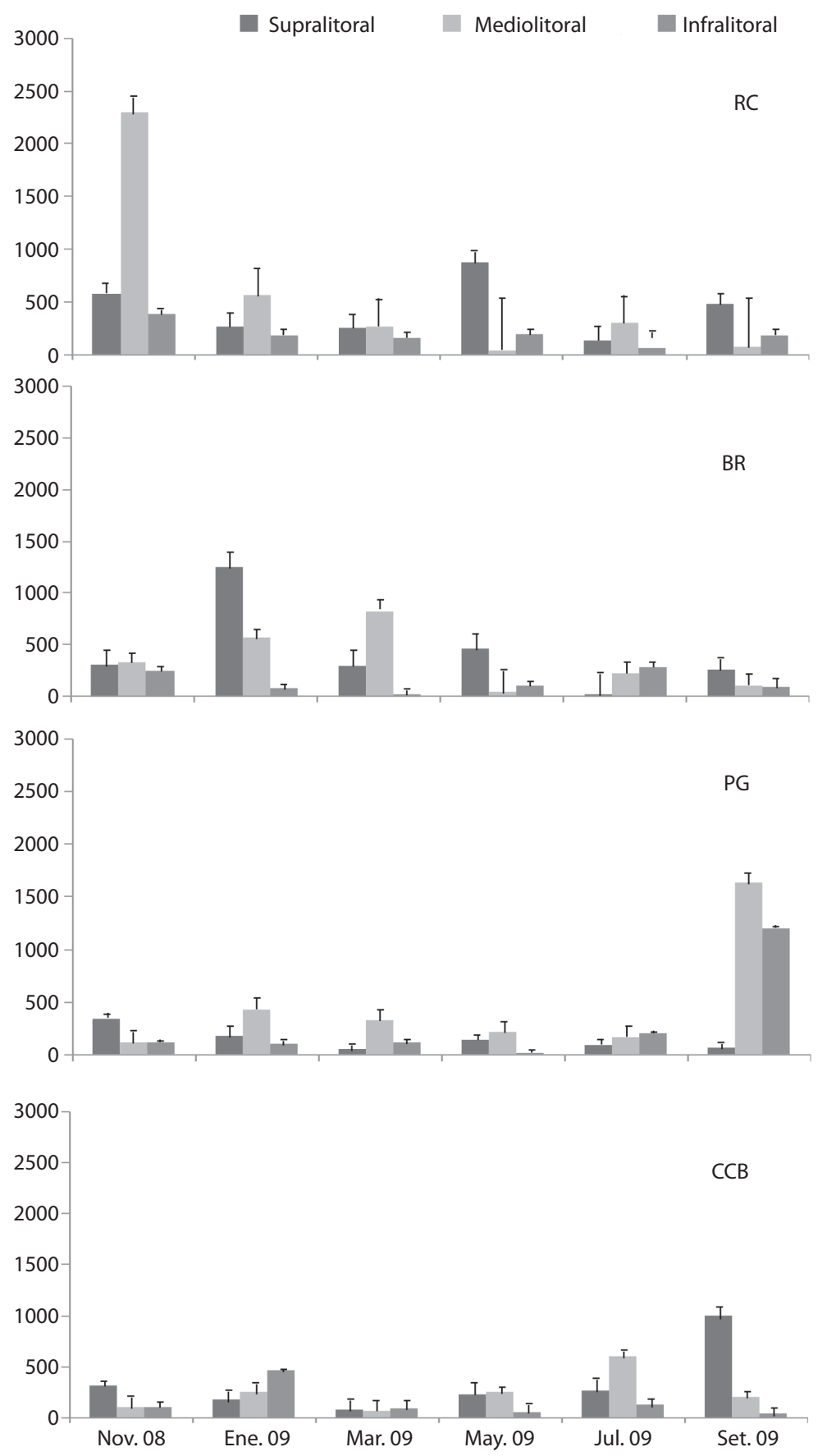

Fig. 2. Abundancia de la macrofauna bentónica entre las zonas supra, medio e infralitoral rocosas de las cuatro localidades (RC: Río Caribe; BC: Boca de Río; PG: Playa Grande, CCB: Copacabana), durante el período de lluvia (may-nov) y sequía (dic-abr).

Fig. 2. Abundance of macrofauna bentonic between areas supra, medium and infralittoral rocky four locations (RC: Río Caribe, BC: Boca Rio, PG: Playa Grande, CCB: Copacabana), during the rainy season (May-November) and dry season (December to April). 
Tanto la diversidad como la equidad, en general presentaron un patrón similar para las localidades estudiadas, encontrándose valores elevados en Boca de Río (3.07bits/ind. y 0.55 , respectivamente), y los más bajos en Playa Grande (2.17bits/ind. y 0.40, respectivamente) para ambos parámetros. Mientras que entre las zonas, en la diversidad como la equidad se obtuvieron máximos valores en la zona infralitoral y mínimos valores en la zona supralitoral (Fig. 3).

Al comparar la abundancia y diversidad durante el período de estudio (lluvia-sequía), estadísticamente no se evidenció diferencias significativas en la macrofauna ( $p>0.05)$, no obstante se observaron diferencias en la abundancia de organismos durante el período de estudio, específicamente en la localidad de Copacabana $(\mathrm{p}=0.049)$.

El análisis ANOSIM-MDS arrojó la formación de dos grupos: a (zona supralitoral y mediolitoral), y b (zona infralitoral), este último grupo indica la similitud a un nivel de estrés de 0.01 (Fig. 4).

\section{DISCUSIÓN}

El número de especies reportadas en esta investigación, es elevado en comparación con otros estudios realizados en costas del litoral centroccidental (Cruz-Motta, 2007), y oriental de Venezuela (Quintana, 1980; Sant \& De Grado, 1997), y fue bajo en comparación con el número de especies señaladas por CAMUDOCA (2006), no obstante, esta última investigación fue realizada en una extensa zona geográfica cercana a las localidades evaluadas en esta investigación.

La distribución de la macrofauna bentónica observada se ajusta al esquema global postulado por Stephenson \& Stephenson (1949), tanto para climas templados como tropicales. En tal sentido, se observaron la presencia de litorínidos y planáxidos, en la zona supralitoral, caracterizados por su gran resistencia ante variables ambientales, coincidiendo con lo descrito por Flores (1973) en Isla de Aves, Fernández (2006) en el estado Sucre, Venezuela, y
Sibaja \& Vargas (2006) en Costa Rica, lo que evidencia que la distribución de estos gasterópodos es constante a nivel mundial.

En la zona mediolitoral, la presencia de los mitílidos concuerda con los resultados obtenidos por León (1997) en Islote Caribe y Lobos, y Fernández \& Jiménez (2007) en costas rocosas del estado Sucre, quienes señalan una franja de representantes de las familias Mytilidae e Isognomidae, los cuales se fijan fuertemente al sustrato mediante filamentos bisales, característica que les facilita alimentarse en esta zona de alta energía, lo que permite el establecimiento de grandes bancos naturales (Buitrago \& Capelo, 1993). La gran abundancia de poliplacóforos observados en este estudio, coincide con las observaciones realizadas por Glynn (1970) quien reporta gran abundancia del género Chiton spp., en costas rocosas intermareales del Caribe.

De igual modo, los balánidos, presentes en la zona mediolitoral, logran adherirse fuertemente a las rocas, peculiaridad observada en los litorales rocosos de todo el mundo. Igualmente, se destaca la presencia de anfípodos, como productores secundarios y terciarios, asociados a otros invertebrados marinos y algas (Tararam, Wakabara, \& Leite, 1986), dependiendo de la interacción con sus depredadores, exposición al oleaje y complejidad del hábitat (Chavanich \& Wilson, 2000). Poco abundantes, pero no menos importantes, fueron los equinodermos, sipuncúlidos y nemertinos presentes en esta zona; resultados similares fueron señalados por CAMUDOCA (2006) en el litoral rocoso de la costa norte del estado Sucre.

En lo que respecta a la zona infralitoral, abundaron los géneros Acmaea, Mitrella, Fissurella, Isognomon, Carditamera, Tegula y Thais, estos últimos, depredadores especializados, que permanecen alimentándose constantemente en zonas sumergidas (Wiley, Circé \& Tunnell, 1982). Los crustáceos (Balanus y Chtamalus), cnidarios (Zoanthus), urocordados (Phallusia nigra), sipuncúlidos, nemertínidos y poliquetos (Sabellidae y Serpulidae) también caracterizaron dicha zona, estos últimos formando arrecifes muchas veces asociados a 
Diversidad H (bits/ind.)
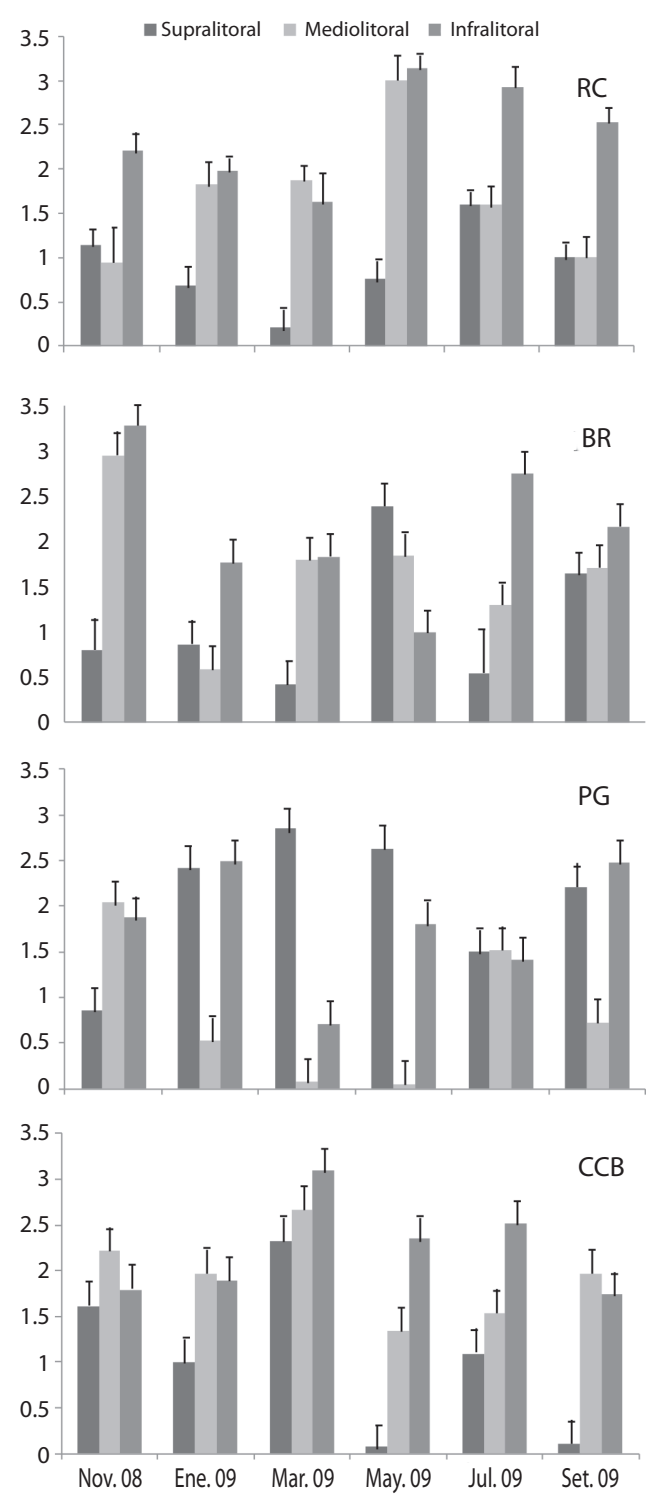

Equidad
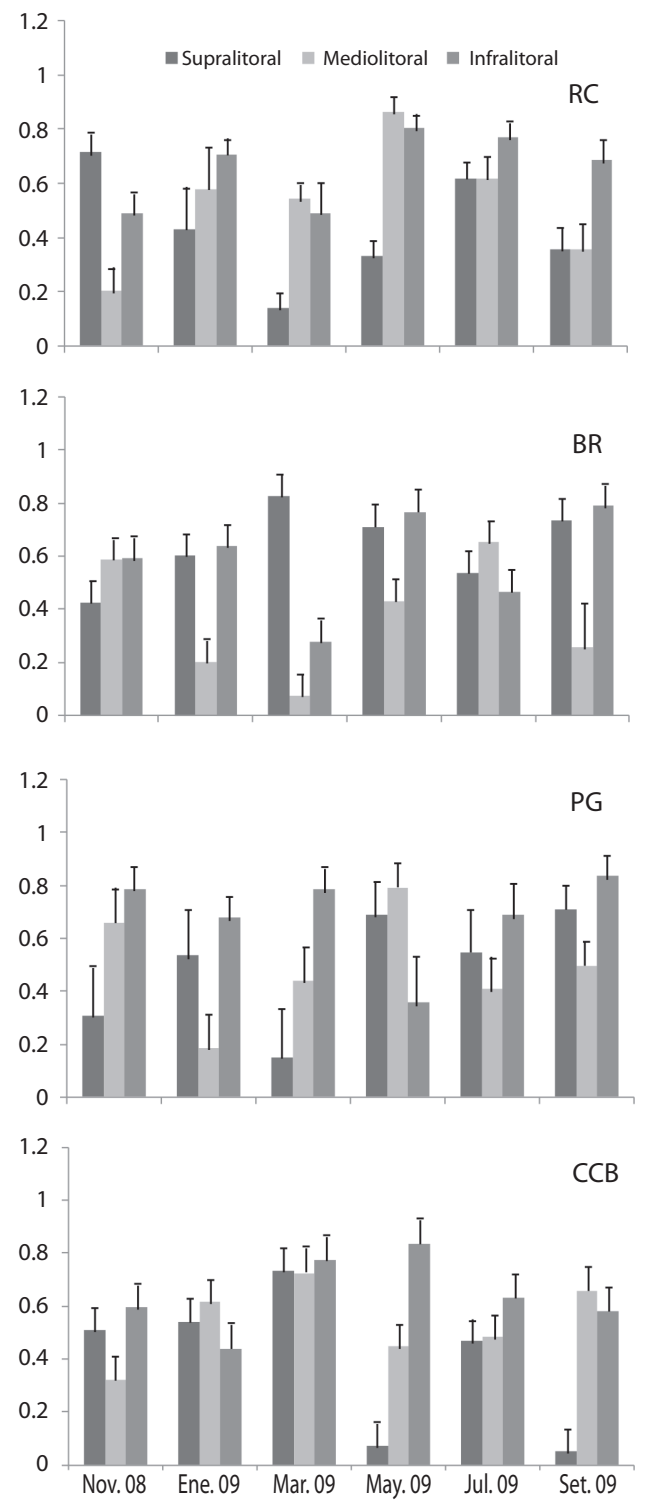

Fig. 3. Diversidad (bits/ind.) y Equidad de la macrofauna bentónica en las zonas supra, medio e infralitoral rocoso de las cuatro localidades (RC: Río Caribe; BC: Boca de Río; PG: Playa Grande y CCB: Copacabana), durante el período de lluvia (may-nov) y sequía (dic-abr).

Fig. 3. Diversity (bits/ind.) and Equity of benthic macrofauna in the areas supra, medium and infralittoral rocky four locations (RC: Río Caribe, BC: Boca Rio, PG: Playa Grande and CCB: Copacabana), during the rainy season (MayNovember) and dry season (December to April). 


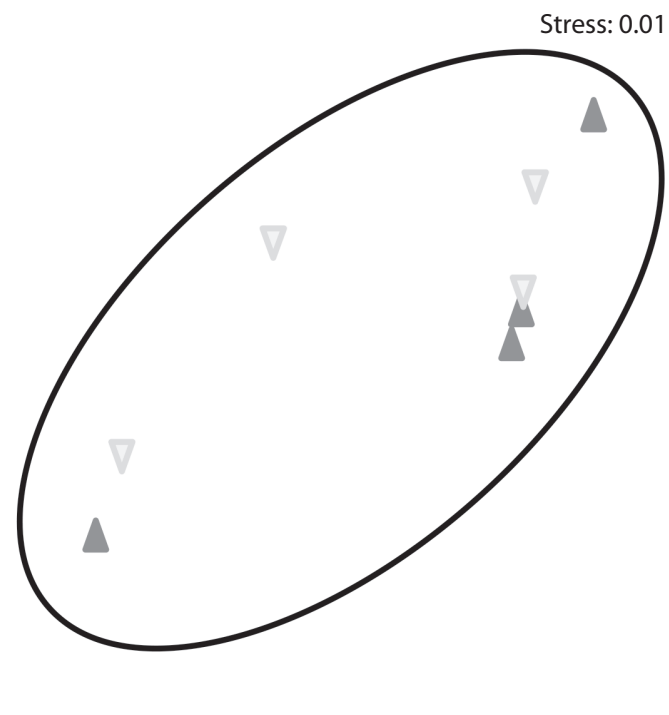

Fig. 4. Diagrama de ANOSIM - MDS de la abundancia de la macrofauna bentónica del litoral rocoso de las zonas (supra, medio e infralitoral) en las cuatro localidades propuestas.

Fig. 4. ANOSIM Diagram - MDS macrofauna abundance of rocky coastline bentonic areas (supra, middle and subtidal) in the four proposed locations.

bancos de bivalvos, que generan microhábitats a otras especies para protegerse y alimentarse (Paredes, Cardoso \& Tarazona, 1999; Zamorano, Moreno \& Duarte, 2000; Sepúlveda, Moreno \& Carrasco, 2003).

Los valores de diversidad y equidad reportados, fueron elevados en contraste con los valores obtenidos por Sant \& De Grado (1997) en el Golfo de Cariaco, no obstante, coincide en que, entre las zonas mediolitoral e infralitoral, los valores fueron mayores, en comparación con la zona supralitoral. Mientras que entre los diferentes grupos taxonómicos, los moluscos fueron los más diversos, lo que concorda con otras investigaciones en la zona oriental del país (Quintana, 1980). En este sentido, Vázquez y Alonso (2004) indican que investigar la diversidad en los sistemas marinos costeros en Venezuela y otras áreas del mundo, ha supuesto un gran desafío científico por la gran extensión de algunas áreas litorales y a veces la poca accesibilidad, lo que sin duda es una limitación práctica para obtener un mayor conocimiento de estos ambientes.
Las diferencias significativas obtenidas en la prueba Kruskal-Wallis, en la abundancia y diversidad de los organismos entre las zonas durante el periodo de estudio, se deben posiblemente a que cada zona expone características muy diferentes y solo se encuentran organismos adaptados a cada una de ellas, lo que se corroboró con la prueba de similitud (MDS). Una posible explicación a esto es que en general, la zona supralitoral y mediolitoral, están expuestas a la emersión continua, y solo existen especies adaptadas a las mismas, capaces de soportar la acción del viento, el oleaje y la desecación, por el contrario, en la zona infralitoral o totalmente sumergida, las condiciones son un poco más estables, de allí la diferencia de abundancia, riqueza y diversidad de especies, lo que se ajusta a lo señalado por Brattström (1985), en la costa rocosa de Panamá.

Si consideramos que en Venezuela existe una extensa zona litoral rocosa, con pocos estudios de invertebrados presentes en ella, no es posible realizar una comparación de dichas comunidades en estos ambientes de alta energía 
y gran incidencia de factores ambientales, por lo que esta investigación servirá de base para el conocimiento de la biodiversidad de tan importante zona del oriente venezolano.

\section{AGRADECIMIENTOS}

Al consejo de investigación UDO- Sucre, por haber aprobado y financiado el proyecto CI. 2 - $030700-1501$ / 08.

\section{RESUMEN}

La zona litoral rocosa esta entre los ambientes físicamente más extremos de la tierra. Los organismos que allí habitan son azotados alternadamente por factores físico-químicos y biológicos. Debido a la gran importancia ecológica que poseen estos ambientes, se estudió la diversidad, abundancia y distribución de la macrofauna bentónica del litoral rocoso, en la costa norte del estado Sucre, Venezuela. Se realizaron muestreos bimensuales, durante un año. La recolecta del material biológico se realizó de forma manual, dentro de una cuadrícula de $0.25 \mathrm{~m}^{2}$, en la zona litoral (supra, medio e infralitoral). Los organismos fueron preservados en formalina al $10 \%$ para su posterior identificación y análisis (parámetros ecológicos y prueba Kruskal-Wallis a la abundancia y diversidad). Se obtuvieron un total 19020 organismos (86spp.), en 8 filos, 45 familias y 47 géneros. Los moluscos fueron los más abundantes y diversos (58 spp.), seguido de los artrópodos (12spp.), anélidos (7spp.), equinodermos (5spp.), y cnidarios, sipuncúlidos, nemertínidos y urocordados (1sp.). Los resultados encontrados, coincidieron con el esquema universal de zonación de Stephenson \& Stephenson (1949). Las localidades de Río Caribe y Boca de Río presentaron los valores más altos de los parámetros ecológicos y Playa Grande los más bajos. Estadísticamente se observaron diferencias en la abundancia y diversidad de la macrofauna, entre las tres zonas. La poca información de la composición y distribución macrobentónica del litoral rocoso, impide una mejor comparación, no obstante los resultados contribuyen al conocimiento de la biodiversidad marina en esta región venezolana.

Palabras clave: bentos, litoral rocoso, ecología, diversidad.

\section{REFERENCIAS}

Abbott, R. (1974). American seashells (2nd ed.). New York, EEUU: Van Nostrand Reinhold Company.

Abbott, R. (1994). Conchas marinas del mundo. México: Trillas.
Abbott, R. \& Morris, P. (1995). Shells of the Atlantic and Gulf Coast and the West Indies. New York, EEUU: Peterson Field Guides.

Belmonte, J. (1985). Contribución al estudio de los equinodermos de la Plataforma Margarita-Coche-Tierra Firme (Trabajo de Grado). Instituto de Tecnología del Mar, Fundación La Salle de Ciencias Naturales. Nueva Esparta, Venezuela.

Brattström, H. (1980). Rocky-shore zonation in the Santa Marta area, Colombia. Sarsia, 65, 163-226.

Brattström, H. (1985). Rocky-shore zonation on the Atlantic coast of Panamá. Sarsia, 70, 179-216.

Buitriago, J. \& Capelo, J. (1993). Los moluscos bentónicos de la región sur-oriental de la Bahía de Pozuelo, Puerto La Cruz, Venezuela. Memorias Sociedad de Ciencia. Naturales La Salle, 52(140), 27-37.

CAMUDOCA. (2006). Estudio de linea base ambiental, socioeconómica y de salud Mariscal Sucre (Informe final). Venezuela: Consultora Ambiental de la Universidad Oriente.

Chavanich, S., \& Wilson, K. (2000). Rocky intertidal zonation of Gammaridean Amphipods in Long island sound, Connecticut. Crustaceana, 73(7), 835-846.

Cruz-Motta, J. (2007). Análisis espacial de las comunidades tropicales intermareales asociadas a los litorales rocosos de Venezuela. Ciencia Mar, 33(2), 133-148.

Damianidis, P., \& Chinthinroglou, C. (2000). Structure and functions of Polychaetofauna living in Mytilus galloprovincialis assemblages in Thermaikos gulf (north Aegean sea). Oceanologica Acta, 23, 110-118.

Dauer, D. (1993). Biological criteria, environmental health and estuarine macrobenthic community structure. Marine Pollution Bulletin, 26, 249-257.

Díaz, J., \& Puyana, M. (1994). Moluscos del Caribe Colombiano. Un catálogo ilustrado. Bogotá: COLCIENCIAS, Fundación Natura e INVEMAR.

Fauchald K. (1977). The polychaete worms. Definitions and keys to the orders, families and genera. Nat. Hist. Mus. Los Angeles City. Sciencie Series, 28, 1-190.

Fernández, J. (2006). Zonación de moluscos del litoral rocoso de la costa sur del golfo de Cariaco y costa norte del estado Sucre, Venezuela (Trabajo de Grado). Universidad de Oriente, Núcleo de Sucre, Venezuela.

Fernández, J., \& Jiménez, M. (2007). Fauna malacológica del litoral rocoso de la costa sur del Golfo de Cariaco y costa norte del estado Sucre, Venezuela. Boletín Instituto Oceanográfico, 46(1), 3-11.

Flores, C. (1973). Notas sobre la distribución horizontal y vertical de los litorinidos (Mollusca: Mesogasteropoda) en aguas costeras de Venezuela. Boletín Instituto Oceanográfico, 12, 67-74.

Glynn, P. (1970). On the ecology of the Caribbean chitons Acanthopleura granulata (Gmelin) and Chiton 
tuberculatus, Linné: density, mortality, feeding, reproduction, and growth. Smithsonian Contribution Zoology, 66, 1-21.

Granadillo, L. (1982). Cirripedos torácicos del oriente venezolano (Trabajo de Grado). Universidad de Oriente, Estado Sucre, Venezuela.

Herrera, A. \& Betancourt, L. (2004). Especies de equinodermos recientes (Echinodermata, Crinoidea, Asteroidea, Ophiuroidea, Echinoidea y Holothurioidea) conocidas para España. Revista Ciencia Sociedad. Instituto Tecnológico de Santo Domingo, 29(3), 506-533.

INVEMAR. (2004). Informe del Estado de los Ambientes Marinos y Costeros en Colombia. Colombia: Litorales rocosos del Caribe.

Jones, W. E. (1980). Field teaching methods in shore ecology. In J. H. Price, D. E. G. Irvine, \& W. H. Farnham (Eds.), The Shore Environment. Vol 1. Methods the Systematics Association Spacial Vol. 17(a) (pp. 19-44). London: Academic Press.

León, L. (1997). Fauna malacológica de los islotes Caribe y Los Lobos. Venezuela: Gobernación del estado Nueva Esparta.

Levinton, J. (1995). Marine Biology: Function, biodiversity, Ecology. New York, Oxford: University press.

Lewis, J. (1961). The fauna of rocky shore of Barbados. Canadian Journal Zoology, 38, 391-435.

Lira, C. (1997). Crustáceos anomuros costeros de la Península de Macanao, Isla de Margarita, Venezuela (Trabajo de Postgrado en Ciencias Marinas). Instituto Oceanográfico de Venezuela, Universidad de Oriente, Cumaná, Venezuela.

Margalef, R. (1995). Ecología. Barcelona, España: Ediciones Omega, S. A.

Martin, J. \& Davis, G. (2001). An updated classification of the recent crustacean, Natural History Museaum of Los Angeles. Country Science Series, 39.

Pachepsky, E., Crawford, J., Brow, J., \& Squire, G. (2001). Towards a general theory of biodiversity. Nature, 410(6831), 923-926.

Paine, R. (1974). Intertidal community structure. Experimental studies on the relationship between a dominant competitor and its principal predador. Oecología, 15, 93-120.

Paredes, C., Cardoso, F., \& Tarazona, J. (1999). Invertebrados del intermareal rocoso de Lima, Perú. Una lista comentada de especies. Revista Peruana de Biología, $6(2), 23-31$.

Quintana, J. (1980). La zonación del litoral rocoso en la región oriental de Venezuela (Trabajo de Postgrado en Ciencias Marinas). Instituto Oceanográfico de Venezuela, Universidad de Oriente, Venezuela.

Ramírez, P. (1997). Islotes Caribe y Los Lobos (Primera edición). Venezuela: Gobernación del Estado Nueva Esparta.
Rodríguez, G. (1959). The marine communities of Margarita Island, Venezuela. Bulletin of Marine Science Gulf and Caribbean, 9, 237-280.

Saiz, J. (1997). Evaluation of adverse biological effects induced by pollution in the Bilbao Estuary (Spain). Journal of Environmental Pollution, 96, 351-359.

Salazar, S., De León, A., \& Salices, H. (1988). Poliquetos (Annelida: Polychaeta) de México. México: Universidad Autónoma de Baja California Sur.

Sant, S. \& De Grado, A. (1997). Zonación de un litoral rocoso en la costa sur del Golfo de Cariaco (Quetepe), Estado Sucre, Venezuela. Saber, 9(1), 69-75.

Sepúlveda, R., Moreno, R., \& Carrasco, F. (2003). Diversidad de Macroinvertebrados asociados a arrecifes de Phragmatopoma moerchi Kinberg, 1867 (Polychaeta: Sabellariidae) en el intermareal rocoso de Cocholgüe, Chile. Gayana, 67(1), 45-54.

Sibaja, J., \& Vargas, J. (2006). Zonación vertical de epifauna y algas en litorales rocosos del Golfo de Nicoya, Costa Rica. Revista. Biología Tropical, 54(1), 49-67.

Stephenson, T., \& Stephenson, H. (1949). Universal feature of zonation between marks on rocky coast. Journal of Ecology, 3(7), 298-306.

Terejova, G., Quintero, A., \& Piñero, A. (1998). Descripción de las características climáticas de Carúpano, estado Sucre (Venezuela). Boletín Instituto Oceanográfico de Venezuela, 37(1,2), 43-52.

Tararam, A., Wakabara, Y., \& Leite, P. (1986). Vertical distribution of amphipods living on algae of brazilian intertidal rocky shores. Crustaceana, 51(2), 183-187.

Uebelacker, J., Johnson, P., \& Victor, B. (1984). Taxonomic guide to the polychaetes of the northern gulf of Mexico. Departament of the interior minerals management service, U.S.

Urosa, J. (1972). Zonificación de un sector de la costa sur del Golfo de Cariaco, Venezuela. Lagena, 30, 3-8.

Vázquez, J. \& Alonso, M. (2004). Ecosistemas marinos costeros del Parque Nacional Bosque Fray Jorge. Chile: Ediciones Universidad de La Serena.

Wiley, G., Circé, R., \& Tunnell, J. (1982). Mollusca of the rocky shores of east central Veracruz state, Mexico. The Nautilus, 96(2), 55-61.

Weinberg, S. (1978). The minimal area problem in invertebrate communities of mediterranean rocky substratc. Marine Biology, 49, 33-40.

Zamorano, J., Moreno, C., \& Duarte, W. (2000). Postsettlement mortality in Phragmatopoma virgini (Polychaeta: Sabellariidae) at the Mehuin Marine Reserve, Chile. Marine Ecology Progress Series, 127(2), 149-155.

Zea, S. (1987). Esponjas del Caribe colombiano (Primera edición). Santa Marta, Colombia: Editorial Catálogo Científico. 